\title{
Thermal conductivity of carbon nanotubes
}

\author{
Jianwei Che, Tahir Çağın and William A Goddard III \\ Materials and Process Simulation Center, Beckman Institute California Institute of \\ Technology, Pasadena, CA 91125, USA \\ E-mail: jiche@caltech.edu
}

Received 2 March 2000

\begin{abstract}
As the sizes of electronic and mechanical devices are decreased to the micron and nanometre level, it becomes particularly important to predict the thermal transport properties of the components. Using molecular level theories, such predictions are particularly important for modelling nano-electronic devices where scaling laws may change substantially but it is most difficult to accurately measure the properties. Hence, using the empirical bond order dependent force field, we have studied here the thermal conductivity of nanotubes' dependence on structure, defects and vacancies. The anisotropic character of the thermal conductivity of the graphite crystal is naturally reflected in the carbon nanotubes. We found that the carbon nanotubes have very high thermal conductivity comparable to diamond crystal and in-plane graphite sheet. In addition, nanotube bundles show very similar properties as graphite crystal in which dramatic difference in thermal conductivities along different crystal axis.
\end{abstract}

\section{Introduction}

The quest to reduce the size of electronic devices and integrated micro/nano-electro-mechanical systems (MEMS and NEMS) provides the main driving force behind the scientific research and technological advancement in nanotechnology. It is now widely accepted that the thermal management in nanosize devices becomes increasingly important as the size of the device reduces. Therefore, the thermal conduction of nanometre materials plays a fundamentally critical role in controlling the performance and stability of nano/micro devices. Among various potential candidates for future MEMS/NEMS applications, carbon nanotubes have a unique position. Their remarkable properties, such as great strength, light weight, special electronic structures and high stability, make carbon nanotubes the ideal material for a wide range of applications. Because of the bright future for carbon nanotubes, a great deal of effort has been devoted to understanding and characterizing their properties [1-4] since the discovery of multi-walled carbon nanotubes by Ijima [5] in 1991 and of single-walled nanotubes by Ijima et al [6] and Bethunes et al [7] in 1993.

Carbon nanotubes have unique electronic properties. They can be either metallic or semiconductor depending on their chirality (i.e. conformational variation). A number of experiments and theoretical investigations have focused on the electronic structures of carbon nanotubes in order to understand the origin of their remarkable phenomena. In addition, significant efforts have been devoted to characterizing their mechanical properties (Young's modulus, energetics). Despite the importance of thermal management in fully functional NEMS/MEMS devices, there has been little progress in characterizing the thermal conduction in nanoscale materials. In this paper we focus on understanding the lattice thermal transport properties of carbon nanotubes. The method employed here [8] is general and can be applied to many other systems.

Due to the technological difficulties of synthesizing high-quality and well-ordered nanotubes, it is still challenging to perform thermal conduction measurements. Thus, it is essential to observe theoretical predictions of the thermal conductivity and the influence of various defects. In general, there are two approaches to calculating the transport properties of materials. One is based on the phenomenological Boltzmann equation (BE) which uses parameters deduced from experiment. The other is based on the fluctuation-dissipation relation from linear response theory which can be produced from first principles. For novel materials where no experimental results are available, the $\mathrm{BE}$ cannot be used to predict transport properties. On the other hand, for molecular dynamics (MD) simulations, the motions of atoms are completely governed by the interatomic interactions described by QM or force field. Thus, with MD the fluctuation-dissipation relation can then be applied directly to calculate the transport coefficients in the linear response regime. Here the only pre-required information is to know the interatomic interactions (force field) for the system. Due to the availability of force field (some from first principles ab initio calculations) for a wide range of systems, it is relativity easy to obtain high-quality empirical potentials from $a b$ initio calculations for simple models and related experimental measurements. Therefore, MD has a unique advantage in predicting the thermal transport properties of 
novel materials and materials on which it is difficult to perform experiments.

In this paper, we use equilibrium MD simulations to calculate carbon nanotube thermal conductivity and its dependence on vacancies and defects. The Green-Kubo $(\mathrm{GK})$ relation derived from linear response theory is used to extract the thermal conductivity from heat current correlation functions. In the next section, we briefly go over the theoretical background for thermal conductivity calculation.

\section{Theoretical background}

The macroscopic thermal conductivity is defined from Fourier's law of heat flow under nonuniform temperature. The steady state heat flow $\vec{J}_{q}$ is obtained by keeping the system and reservoirs in contact,

$$
\vec{J}_{q}=-\Lambda \nabla T
$$

where $\Lambda$ is called the thermal conductivity tensor, and $\vec{J}_{q}$ is the heat current produced by the temperature gradient $\nabla T$. Fourier's law of heat flow can be derived from linear response theory [9]. For isotropic systems, the conventional thermal conductivity $\lambda$ is given by the average quantity of different directions,

$$
\lambda=\frac{1}{3} \operatorname{Tr} \Lambda .
$$

Note that the thermal conductivity calculated here does not include the electronic contribution and no net particle flow persists in the system. The contribution to the thermal conduction only comes from the atomic vibrations, so-called lattice thermal conductivity. In MD simulations, the heat current is given by the following equations:

$$
\begin{gathered}
\vec{R}=\sum_{i} \vec{r}_{i} h_{i} \\
\vec{J}_{q}=\frac{\mathrm{d}}{\mathrm{d} t} \vec{R},
\end{gathered}
$$

where $h_{i}$ is the total energy of particle $i$, which includes kinetic and potential energies. For pairwise interactions, it is

$$
h_{i}=\frac{p_{i}^{2}}{2 m_{i}}+\frac{1}{2} \sum_{j} V_{i j} .
$$

Following the fluctuation-dissipation theorem, the thermal conductivity tensor can be expressed in terms of heat current correlation functions [9],

$$
\Lambda=\frac{1}{k_{B} T^{2} V} \int_{0}^{\infty} \mathrm{d} t C_{J J}^{q}(t)
$$

where

$$
C_{J J}^{q}(t)=\left\langle\vec{J}_{q}(t) ; \vec{J}_{q}(0)\right\rangle
$$

where $C_{J J}^{q}$ is called the quantum canonical correlation function and $\langle a ; b\rangle$ is defined as

$$
\langle a ; b\rangle=\frac{1}{\beta} \int_{0}^{\beta} \mathrm{d} \xi \operatorname{Tr}[\rho \exp (\xi H) a \exp (-\xi H) b],
$$

and $\rho$ is the density matrix of the system at equilibrium; $a, b$ are dynamic operators.
$C_{J J}^{q}$ is a quantum mechanical correlation function; it is usually difficult to evaluate directly. Here, we only briefly discuss its relationship with its classical counterpart. In the classical limit, the Planck constant $\hbar$ approaches zero and the canonical correlation reduced to the usual classical correlation function. The classical counterparts of (6) and (7) are

$$
\begin{gathered}
\Lambda=\frac{1}{k_{B} T^{2} V} \int_{0}^{\infty} \mathrm{d} t C_{J J}^{c}(t) \\
C_{J J}^{c}(t)=\left\langle\vec{J}_{q}(t) \vec{J}_{q}(0)\right\rangle
\end{gathered}
$$

where $C_{J J}^{c}(t)$ is the classical correlation function given by the phase space averaging,

$$
\langle a b\rangle=\frac{\int \mathrm{d} \Gamma \exp (-\beta H) a b}{\int \mathrm{d} \Gamma \exp (-\beta H)} .
$$

Both classical and canonical correlation functions are symmetric. Naturally, quantum effects are not important when $T \gg T_{D}$, where $T_{D}$ is the Debye temperature. However, when the above inequality is not satisfied, certain cautions are needed when replacing quantum canonical correlation functions by classical correlation functions. In order to estimate the quantum correction to the classical equation, we ought to find the relationship between the quantum canonical correlation function and its classical counterpart. Here, we employed a harmonic analysis to examine the quantum correlation function [8]. A symmetrized quantum mechanical correlation function can be defined based on a quantum anticommutator, i.e.

$$
C^{\prime}(t)=\frac{\left\langle[\mathrm{O}(t), \mathrm{O}(0)]_{+}\right\rangle}{2} .
$$

After some algebra [8], the relation between the quantum canonical correlation function and the symmetrized quantum correlation function can be obtained:

$$
\tilde{C}_{J J}^{q}(\omega)=\frac{2}{\beta \hbar \omega} \tan h \frac{\beta \hbar \omega}{2} \tilde{C}^{\prime}(\omega)
$$

where the tilde represents the Fourier transform of the respective functions. To further examine the quantum effects, it is useful to generalize the definition of thermal conductivity to include the frequency dependence. The generalized thermal conductivity is then written as

$$
\begin{aligned}
\Lambda(\omega) & =\frac{1}{2 k_{B} T^{2} V} \int_{-\infty}^{\infty} C_{J J}^{q}(t) \mathrm{e}^{\mathrm{i} \omega t} \mathrm{~d} t \\
& =\frac{1}{2 k_{B} T^{2} V} \tilde{C}_{J J}^{q}(\omega) .
\end{aligned}
$$

Substituting (13) into (14), leads to

$$
\Lambda(\omega)=\frac{1}{2 k_{B} T^{2} V} \frac{2}{\beta \hbar \omega} \tan h \frac{\beta \hbar \omega}{2} \tilde{C}^{\prime}(\omega) .
$$

Until now, all the derivations are exact. To establish the relationship between $C^{\prime}$ and classical correlation function $C^{c}$, there is no general way. Here, we make use of the harmonic mapping method, which is exact only for harmonic systems,

$$
\begin{aligned}
\tilde{C}^{\prime}(\omega) & =\frac{1}{2}\left[\tilde{C}^{+}(\omega)+\tilde{C}^{-}(\omega)\right] \\
& =\frac{\beta \hbar \omega}{2} \operatorname{coth} \frac{\beta \hbar \omega}{2} \tilde{C}^{c}(\omega) .
\end{aligned}
$$


Substituting (16) into (15), leads to

$$
\Lambda(\omega)=\frac{1}{2 k_{B} T^{2} V} \tilde{C}_{J J}^{c}(\omega) .
$$

This is just the classical thermal conductivity

$$
\Lambda(0)=\Lambda_{c}
$$

This result is expected since (16) is only exact for harmonic systems. In harmonic systems, each normal mode is decoupled from others. Therefore, the only contribution to thermal conductivity for a macroscopic system is from phonon modes that have wavelength equal to or longer than the macroscopic length scale, which translates into effectively zero-frequency mode. As pointed out in our previous work [8], this is very similar to vibrational relaxation of a harmonic system bilinearly coupled to a harmonic bath, where both the full quantum relaxation rate and full classical relaxation rate are the same.

However, the major contribution to thermal conductivity of a system is the anharmonicity in the interaction potential. Because of the anharmonicity, phonons can scatter from each other, which gives rise to the limited phonon mean free path and finite thermal conductivity. Although the mapping equation (i.e. (16)) is only exact for harmonic systems, the classical correlation function already includes the anharmonic effects when it is evaluated during MD simulations. In this sense, apart from the approximate nature of the prefactor in (15), the quantum correlation function obtained from the mapping can still capture the dominant anharmonic effects. Based on the above reasoning, we carry out the thermal conductivity calculation using only classical correlation functions.

\section{Numerical calculations}

The empirical interatomic interaction used in our calculations is the Brenner-type of bond order dependent potential [10, 11]. The Brenner potential is parametrized for hydrocarbon systems, and is widely used in modelling carbon based systems, such as diamond, graphite sheet, fullerenes and carbon nanotubes. In all MD simulations, 1 fs time step is employed, and $40 \mathrm{ps}$ initial MD is used to equilibrate the systems. After equilibration, 400 ps constant energy (NVE) simulation is carried out, and the heat current is calculated every time step. The average temperature in all simulations is $300 \mathrm{~K}$.

One main concern of using MD to calculate the thermal conductivity is the size effect of the simulation box due to periodic boundary conditions. When the simulation is conducted in a small box, phonons will get scattered more frequently because they re-enter the simulation box before they can be dissipated. In other words, the mean free path of phonons is limited to the order of the simulation box. This artifact usually underestimates the thermal conductivity. In order to obtain the correct thermal conductivity, we test the convergence of MD simulations on thermal conductivity by using different size systems and early time correlation function analysis [8]. For a $(10,10)$ single-walled nanotube, four different systems are investigated. They contain 400,

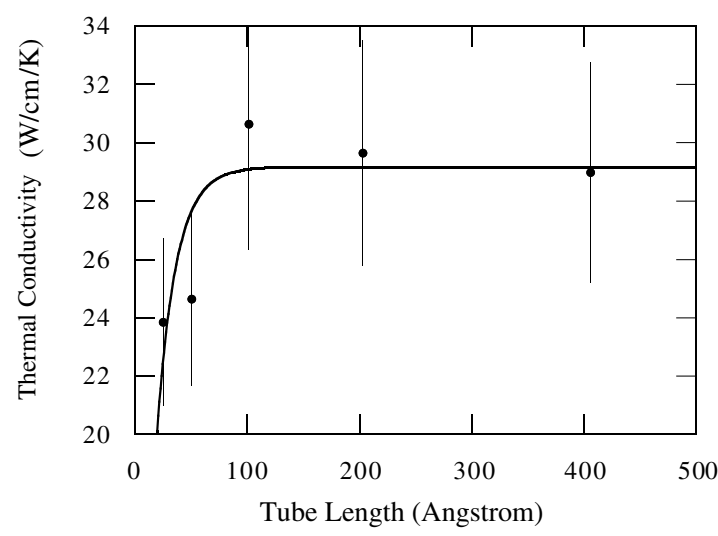

Figure 1. Convergence of thermal conductivity.

$800,1600,3200$ and 6400 atoms, respectively. As we expected, the phonon mean free path is the limiting factor to obtain accurate results. For a small simulation system, the calculated thermal conductivity is smaller than the correct value because of the overestimation of phonon scattering. As the simulation system size gets larger, the theoretical value converges to a constant which is independent of simulation size. The convergence behaviour can be seen in figure 1 . The theoretical predicted value approaches $29.8 \mathrm{~W} \mathrm{~cm}^{-1} \mathrm{~K}^{-1}$ along the tube axis, which is very high in comparison with conventional materials. We also mention here that the thermal conductivity for an isolated single-wall carbon nanotube is not a well defined quantity, since the cross section of heat conduction can be chosen in various ways. In this paper, we choose a $1 \AA$ thickness cylinder as the geometric configuration. Other choices can also be made such as choosing the van de Waals thickness. The choice of the cross section is not important here since we will make comparison under consistent conditions. Later in this section, we will show the thermal conductivity for nanotube bundles where the cross section is uniquely defined, and the results can be used to compare with experiments and other materials.

In figure 2 we show the initial autocorrelation function of the heat current along the tube axis for the system containing 3200 atoms. The figure clearly shows very fast decay at the beginning followed by a very slow decay. The fast decay is due to the optical modes in the nanotube that have high vibrational frequency. This behaviour also indicates that the high vibrational modes do not contribute to the thermal conductivity in a significant way [8]. The correlation function can be well characterized by a double exponential function with two time constants.

The high value of thermal conductivity is for a pure and defect-free carbon nanotube. However, nanotubes can have natural defects and vacancies. It is therefore important to know how defects influence the thermal conduction properties of carbon nanotubes. Unlike macroscopic threedimensional materials, carbon nanotubes can be thought of as a quasi one-dimensional wire. In figure 3, the thermal conductivities are calculated for various vacancy concentrations. As we expected, the thermal conductivity decreases as the vacancy concentration increases. However, the rate of decrease in thermal conductivity is quite unexpected. We have calculated the vacancy influence in 


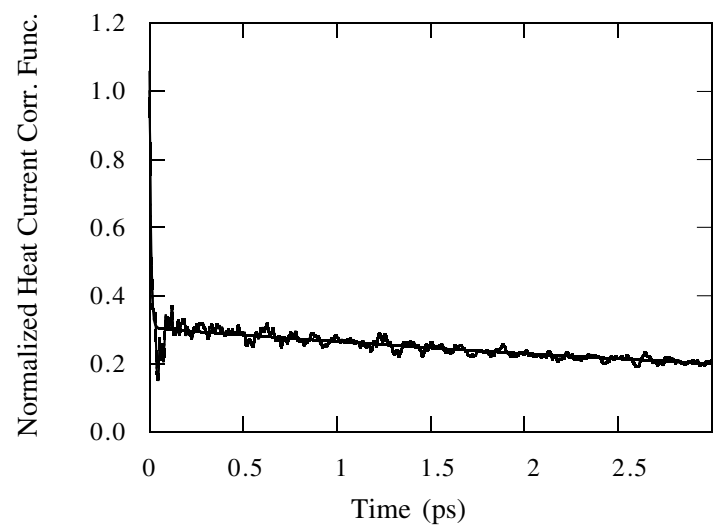

Figure 2. Current autocorrelation function.

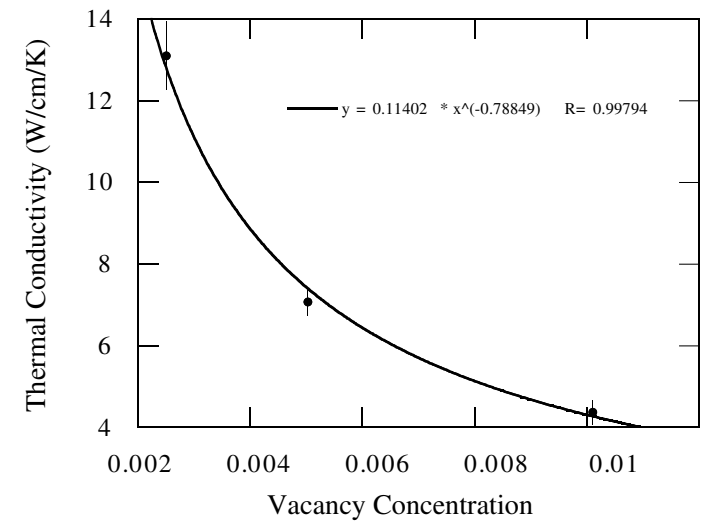

Figure 3. Thermal conductivity as a function of vacancy concentration.

thermal conductivity for diamond crystal previously [8], which also showed an inverse power law relation (exponent $\alpha=0.69$ ) between thermal conductivity and vacancy concentration. It is natural to think that vacancies should have much severer effects in one-dimensional materials than in three-dimensional ones. However, the calculation results show that vacancies in nanotubes are not much more influential than in three-dimensional diamond. This is surprising, and is probably due to the fact that the strong valence double bond network provides effective additional channels for phonons to bypass the vacancy sites. The detailed mechanism needs to be studied further.

Similarly, conformational defects can also reduce the thermal conductivity significantly. One of the common conformation defects in nanotubes is $(5,7,7,5)$ defect where four hexagons change into two pentagons and two hexagons. Figure 4 shows how this conformational defect affects the overall thermal conductivity of nanotubes. Compared with vacancies, the $(5,7,7,5)$ defect is a milder form of point defect, since it does not change the basic bonding characteristic and causes much less overall structural deformation. So both the rate and absolute amount of decrease in thermal conductivity here are less than in the case of vacancies.

As we mentioned earlier, the absolute value of an isolated single-wall nanotube is ambiguous because the heat conduction cross-section is not uniquely defined. However,

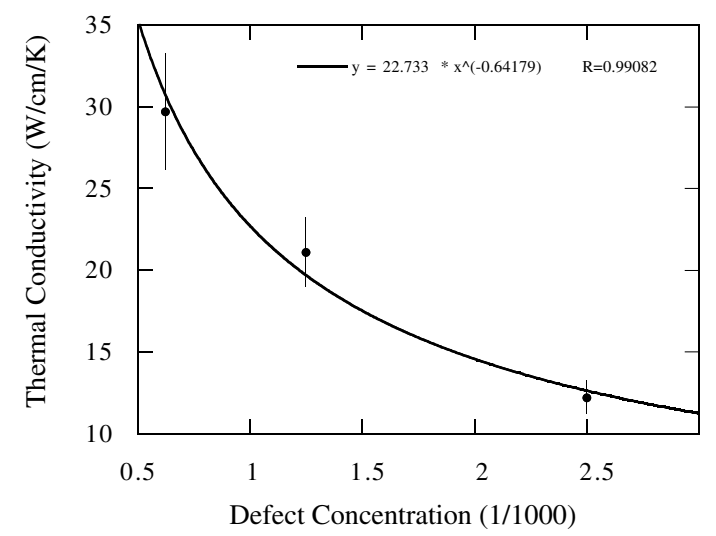

Figure 4. Thermal conductivity as a function of defect concentration.

this problem can be avoided in nanotube bundles. In this case, the cross section can be well defined and it is unique. In addition, nanotube bundles are materials that can be measured unambiguously in real experiments. Therefore, we also carried out the thermal conductivity calculation for $(10,10)$ nanotube bundles in close packing condition. The simulations show that the nanotube bundle has very high thermal conductivity along the tube axis, $9.5 \mathrm{~W} \mathrm{~cm}^{-1} \mathrm{~K}^{-1}$, which is comparable to simulated graphite in-plane thermal conductivity, $10 \mathrm{~W} \mathrm{~cm}^{-1} \mathrm{~K}^{-1}$. In the direction perpendicular to the tube, the nanotube bundle's thermal conductivity is much lower, $0.056 \mathrm{~W} \mathrm{~cm}^{-1} \mathrm{~K}^{-1}$, which is also similar to simulated graphite out-of-plane thermal conductivity, $0.055 \mathrm{~W} \mathrm{~cm} \mathrm{~K}^{-1} \mathrm{~K}^{-1}$. Note that the in-plane graphite thermal conductivity is very different from experimental values, because the interaction potential used here has much larger anharmonicity than a real system. Detailed analysis of the potential anharmonicity can be found in [8]. Nevertheless, we believe that the relative relation between graphite and nanotube bundles still holds. The well ordered carbon nanotube bundles have similar thermal conductivity to graphite both in plane and out of plane.

\section{Conclusions}

We have reported here the high thermal conductivity for single-walled nanotubes based on MD simulations. The implication is very important since it promises efficient thermal management in nanotube-based MEMS/NEMS devices. The defect influence on the thermal conductivity of carbon nanotube reveals an interesting phenomenon that has not been noted before. More detailed analysis is needed to understand the origin of this behaviour. In addition, more accurate interaction potentials will be developed to quantitatively study the nanotubes and other nanostructures.

\section{Acknowledgments}

This research was funded by a grant from DOE-ASCI. The facilities of the MSC are also supported by grants from NSF (ASC 92-17368 and CHE 91-12279), ARO (MURI), ARO (DURIP), ONR (DURIP), Chevron Petroleum Technology 
Co., Asahi Chemical, Owens-Corning, Exxon, Chevron Chemical Co., Asahi Glass, Chevron Research Technology Co., Avery Dennison, BP America, and the Beckman Institute.

\section{References}

[1] Chico L, Crespi V H and Benedict L X 1996 Phys. Rev. Lett. 76971

[2] Langer L, Bayot V and Grivei E 1996 Phys. Rev. Lett. 76479

[3] Ebbesen T W, Lezec H J, Hiura H, Bennett J W, Ghaemi H F and Thio T 1996 Nature 38254
[4] Pichler T, Knupfer M and Golden M S 1998 Phys. Rev. Lett. 804729

[5] Iijima S 1991 Nature 35456

[6] Iijima S and Ichihashi T 1993 Nature 363603

[7] Bethunes D S, Kiang C H, Devries M S, Gorman G, Savoy R, Vazquez J and Beyers R 1993 Nature 363605

[8] Che J, Çağın T and Goddard W A III 2000 J. Chem. Phys. submitted

[9] Kubo R, Toda M and Hashitsume N 1985 Statistical Physics vol 2 (Berlin: Springer)

[10] Brenner D W 1990 Phys. Rev. B 429458

[11] Che J, Çağın T and Goddard W A III 1999 Theor. Chem. Acct. 102346 